\title{
Evaluation of bond strength of silorane and methacrylate based restorative systems to dentin using different cavity models
}

\author{
Stephano Zerlottini ISAAC, Ana Claudia Pietrobom BERGAMIN, Cecília Pedroso TURSSI, Flávia Lucisano Botelho \\ do AMARAL, Roberta Tarkany BASTING, Fabiana Mantovani Gomes FRANÇA
}

1- Department of Restorative Dentistry, São Leopoldo Mandic Institute and Research Center, Campinas, SP, Brazil.

\begin{abstract}
Corresponding address: Fabiana Mantovani Gomes França - Instituto e Centro de Pesquisas Odontológicas São Leopoldo Mandic - Departamento de Odontologia Restauradora - Dentística - Rua José Rocha Junqueira -13 - Swift - Campinas - SP - 13045-755 - Brasil - Phone/Fax: 55-19-3211-3600 - ramal 249 - e-mail: biagomes@yahoo.com
\end{abstract}

Submitted: February 6, 2013 - Modification: May 6, 2013 - Accepted: August 12, 2013

\section{ABSTRACT}

\begin{abstract}
$\mathrm{O}$ bjectives: The aim of this in vitro study was to evaluate the microtensile bond strength ( $\mu$ TBS) to dentin of two different restorative systems: silorane-based (P90), and methacrylate-based (P60), using two cavity models. Material and Methods: Occlusal enamel of 40 human third molars was removed to expose flat dentin surface. Class I cavities with $4 \mathrm{~mm}$ mesial-distal width, $3 \mathrm{~mm}$ buccal-lingual width and $3 \mathrm{~mm}$ depth (C-factor=4.5) were prepared in 20 teeth, which were divided into two groups $(n=10)$ restored with P60 and P90, bulk-filled after dentin treatment according to manufacturer's instructions. Flat buccal dentin surfaces were prepared in the 20 remaining teeth $(C$-factor $=0.2)$ and restored with resin blocks measuring $4 \times 3 \times 3 \mathrm{~mm}$ using the two restorative systems $(n=10)$. The teeth were sectioned into samples with area between 0.85 and $1.25 \mathrm{~mm}^{2}$ that were submitted to $\mu$ TBS testing, using a universal testing machine (EMIC) at speed of 0.5 $\mathrm{mm} / \mathrm{min}$. Fractured specimens were analyzed under stereomicroscope and categorized according to fracture pattern. Data were analyzed using ANOVA and Tukey Kramer tests. Results: For flat surfaces, P60 obtained higher bond strength values compared with P90. However, for Class I cavities, P60 showed significant reduction in bond strength $(p<0.05)$. No statistical difference between restorative systems was shown for Class I cavity model ( $p>0.05$ ), or between Class I Cavity and Flat Surface group, considering P90 restorative system $(p>0.05)$. Regarding fracture pattern, there was no statistical difference among groups ( $p=0.0713$ ) and $56.3 \%$ of the fractures were adhesive. Conclusion: It was concluded that methacrylate-based composite $\mu$ TBS was influenced by cavity models, and the use of silorane-based composite led to similar bond strength values compared to the methacrylatebased composite in cavities with high C-factor.
\end{abstract}

Keywords: Composite resins. Dental cavity preparation. Tensile strength.

\section{INTRODUCTION}

The majority of composites used in restorative dentistry are chemically based on the polymerization reaction of methacrylates. This reaction presupposes the conversion of monomer molecules into a polymeric network, within which van der Waals spaces are replaced by covalent bonds that approximate the molecules and cause considerable contraction of the composite ${ }^{26}$.

Polymerization shrinkage is an intrinsic property of the resin matrix ${ }^{18}$, which has been undesirably ascribed to problems associated with failures in posterior teeth resin composite restorations. These failures may be cohesive fracture of enamel prisms and microgap formation, with rupture of adhesive bonds and consequent withdrawal of the restorative material from the cavity walls. Consequently, marginal microleakage, marginal staining, postoperative sensitivity and secondary caries can be expected ${ }^{4,28}$.

The magnitude of the stress generated by 
polymerization shrinkage depends on several factors, such as the composite modulus of elasticity ${ }^{26}$, molecule size, the relationship between filler volumetric percentage and organic matrix percentage of, amount of functionally reactive methacrylate groups per monomer, extension, depth and speed of polymerization and the cavity configuration factor (C-factor $)^{8}$. The C-factor, determined by the ratio between the area of adhered surfaces and the area of free surfaces, has a direct relationship with the capacity to release the stresses generated during polymerization. Choi, et al. ${ }^{5}$ (2004) affirmed that the greater the C-factor was, the lower would be the bond strength due to the greater stress generated in the tooth structure at the bond interface. The elastic modulus of the composite is another important factor to consider. In general, composites with higher elasticity modulus show less internal deformation and, therefore, may have less capacity of compensating the stress generated by polymerization shrinkage, because they are unable to absorb part of the energy that reaches the bond interface ${ }^{8,17}$. The degree of conversion of monomers into polymers also has an influence: the higher the degree, the more contraction there will be ${ }^{8}$. On the other hand, high degree of conversion, which can be achieved with adequate polymerization, generally leads to better physical-chemical properties of resin composites. Therefore, a technical dilemma is presented: polymerization shrinkage and mechanical properties are antagonistic properties ${ }^{10}$.

With this respect, alternatives have been created to minimize the undesirable effects of polymerization shrinkage: insertion and light activation techniques ${ }^{8,20}$, development of adhesives capable of acting as more efficient and resistant interfaces between dental tissues and resin composites ${ }^{24}$ and the development of resins with a low degree of polymerization shrinkage9,23,27.

The chemical basis of the new generation of composites is the replacement of the conventional methacrylate-based organic matrix with a matrix derived from a molecule denominated silorane, whose polymerization reaction occurs by opening of a cationic ring before the formation of cross links $6,19,27$. This opening would supposedly partially compensate the volumetric reduction that takes place when crosslinking occurs. On conclusion of the polymerization reaction, the contraction observed would be less than $1 \%{ }^{27}$ against 1.5 to $6 \%$ of contraction observed in methacrylate based composites ${ }^{5}$. It is important to mention that the silorane-based composites require a specific adhesive system, composed of two phases: a self-etching hydrophilic primer and a hydrophobic adhesive resin, based on methacrylate chemistry and, therefore, compatible with the resin systems derived from methacrylate ${ }^{2}$. There is evidence of less degradation of the silorane bond interface due to its highly hydrophobic properties 2,16,25.

In view of the foregoing discussion, the aim of this study was to evaluate, by means of the microtensile bond strength test, the bond strength of restorative systems derived from methacrylate and silorane, in two cavity models: Class I cavity and Flat Surface. The null hypothesis tested was that the restorative systems and the cavity models would not influence bond strength to dentin.

\section{MATERIAL AND METHODS}

\section{Ethical aspects}

The present study was approved by the local Ethics Committee (2010/0295).

\section{Experimental design}

The factors under study were: 1) Restorative systems, at two levels: Single Bond + P60 (methacrylate); Silorane Adhesive System + P90 (silorane); 2) Cavity models at two levels: Flat Surface (C-factor=0.2) and Class I cavity (C-factor=4.5).

The experimental units consisted of 40 human third molars, randomly distributed into four experimental groups: G1 - P60 Flat Surface; G2 P60 Class I; G3 - P90 Flat Surface; G4 - P90 Class I. After the teeth were restored, longitudinal stickshaped sections were obtained, which constituted replicas. The response variable, of the continuous quantitative type, was bond strength measured by the microtensile technique (in $\mathrm{MPa}$ ). The mean of the replicas of each specimen, i.e., the sections, was considered the value of each specimen.

Figure 1 describes the materials used in the experiment. Figure 2 illustrates the restorative steps.

Forty third molars, free of caries lesions, stains or cracks were selected $(n=10)$, which were obtained from the tooth bank of the São Leopoldo Mandic Institute and Research Center. The teeth were stored in a $0.1 \%$ thymol solution for up to three months before being used, then they were cleaned with periodontal curettes (Duflex - SS White Artigos Dentários Ltda., Rio de Janeiro, RJ, Brazil) and stored in physiological solution at 37 degrees for 24 hours.

\section{Cavity preparations}

The Class I cavity model involved sectioning in the horizontal direction at the height of the central sulcus to expose middle dentin and obtain a smooth surface with the use of a double-faced diamond disc (KG \#7020/ KG Sorensen Ind. e Com. Ltda., Cotia, SP, Brazil), under cooling. The dentin surface was abraded with 600 grit silicon carbide abrasive 
paper (Carborundum Abrasivos S/A, Vinhedo, SP, Brazil) in a water-cooled polishing machine (APL-4, Arotec S/A, São Paulo, SP, Brazil), to regularize it. After this procedure, Class I cavities were prepared using a cylindrical diamond tip with a rounded end KG\#3145 (KG Sorensen Ind. e Com. Ltda., Cotia, $\mathrm{SP}$, Brazil).

A maximum number of five teeth were prepared per diamond tip, in order to prevent the loss of granulation occurring during use from leaving different groove patterns on the dentin surface. With the purpose of standardizing the cavity preparations, which were performed by the same operator, a digital caliper (Mitutoyo Sulamericana Ltda., Suzano, SP, Brazil) was used to measure the cavity dimensions, accepting those cavities with a variability of $\pm 0.1 \mathrm{~mm}$. The cavities were made with high speed turbines (Kavo do Brasil Ltda, Joinville, SC, Brazil), cooled by a jet of air/water and the mean dimensions established were: mesio-distal length of $4 \mathrm{~mm}$, depth of $3 \mathrm{~mm}$ and vestibularlingual height of $3 \mathrm{~mm}$, which configured a cavity with a high C-factor.

The cavities obtained were restored with methacrylate-based composites (Filtek P-60, 3M Dental Products, St. Paul, MN, USA), and siloranebased (Filtek P-90, 3M Dental Products, St. Paul, MN, USA), after application and light activation of their respective adhesive systems (according to the manufacturers' instructions - Figure 1).

\begin{tabular}{|c|c|c|}
\hline $\begin{array}{l}\text { Material/Manufacturer } \\
\text { Batch number }\end{array}$ & Main composition & Instructions for use \\
\hline $\begin{array}{l}\text { Composite Filtek P60 } \\
\text { Shade A3 } \\
3 \text { M ESPE Dental Products, } \\
\text { MN, USA } \\
\text { N127655 }\end{array}$ & \begin{tabular}{|l} 
Bis-GMA \\
Bis-EMA \\
UDMA \\
Zirconium and silica particles $(61 \%$ by volume $)$ \\
with sizes between 0.01 and $3.5 \mu \mathrm{m}$.
\end{tabular} & $\begin{array}{l}\text { Maximum increment of } 2.5 \mathrm{~mm} \text {, } \\
\text { inserted in the cavity and light activated } \\
\text { for } 40 \mathrm{~s} \text {. }\end{array}$ \\
\hline $\begin{array}{l}\text { Composite Filtek P90 } \\
\text { Shade A3 } \\
\text { 3M ESPE Dental Products, } \\
\text { MN, USA } \\
\text { N130928 }\end{array}$ & $\begin{array}{l}\text { 5-15\% of 3, 4-epoxy cyclohexyl cyclopolymethyl } \\
\text { siloxane; } \\
5-15 \% \text { of 3, 4-epoxy cyclohexyl cyclopolymethyl } \\
\text { siloxane; } \\
50-70 \% \quad \text { silanized quartz;10-20\% Yttrium } \\
\text { fluoride; } \\
\text { camphorquinone. }\end{array}$ & $\begin{array}{l}\text { Maximum increment of } 2.5 \mathrm{~mm} \text {, } \\
\text { inserted in the cavity and light activated } \\
\text { for } 40 \mathrm{~s} \text {. }\end{array}$ \\
\hline $\begin{array}{l}\text { Adper Single Bond } 2 \text { Adhesive } \\
\text { 3M ESPE Dental Products, } \\
\text { MN, USA } \\
\text { CCBR }\end{array}$ & $\begin{array}{l}\text { Bis-GMA, HEMA, dimethacrylate ethanol, water, } \\
\text { photoinitiators, nanoparticles, compolymer of } \\
\text { methacrylic acid }(10 \%) \text {. }\end{array}$ & $\begin{array}{l}\text { Acid etching for } 15 \mathrm{~s} \text {. Rinsing for } 10 \mathrm{~s} \text {. } \\
\text { Drying with air for } 2 \mathrm{~s} \text {. } \\
\text { Application of two consecutive layers } \\
\text { of adhesive. } \\
\text { Drying for } 5 \mathrm{~s} \text {. } \\
\text { Light activation for } 20 \mathrm{~s} \text {. }\end{array}$ \\
\hline $\begin{array}{l}\text { Silorane Adhesive System } \\
\text { 3M ESPE Dental Products, } \\
\text { MN, USA } \\
\text { 9BM/9BK }\end{array}$ & 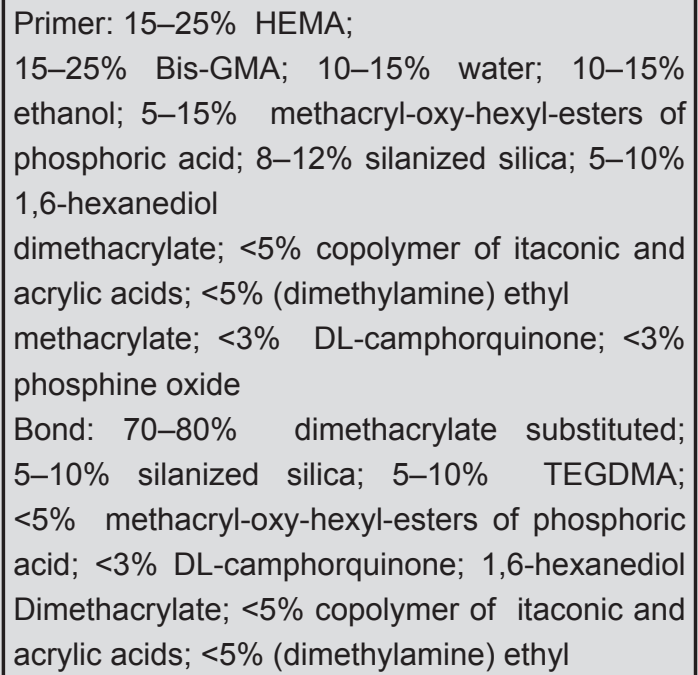 & $\begin{array}{l}\text { Active Primer application for } 15 \\
\text { seconds. Drying with air. Light } \\
\text { activation for } 10 \mathrm{~s} \text {. Drying with air. } \\
\text { Application of bond. Light activation } \\
\text { for } 10 \mathrm{~s} \text {. }\end{array}$ \\
\hline
\end{tabular}

Figure 1- Lots, manufacturers, main components and instructions for use of resin composites and adhesive systems used 
The composites were inserted in a single increment? ${ }^{7}$, and after filling the cavities, a transparent matrix strip (polyester strip, TDV Dental Ltda., Pomerode, SC, Brazil) was placed over the restorations and a $250 \mathrm{~g}$ weight was placed on the set for 30 seconds. When this time had elapsed, the weight was removed and light activation was performed in continuous mode for 40 seconds, using light provided by a Halogen lamp unit (Demetron LC, Kerr Manufacturing Company, Orange, CA, USA), with a mean irradiance of $450 \mathrm{~mW} / \mathrm{cm}^{2}$ controlled with a radiometer (RD-7 Ecel Ind. e Com. Ltda., Ribeirão Preto, SP, Brazil), measured after each two cycles of 40 seconds, the average irradiance was $450 \mathrm{~mW} / \mathrm{cm}^{2}$.

The Flat Surface cavity model served as the control group, as the possible negative influences arising from the high C-factor in the Class I model were not expected to occur in the control group specimens. For this, Class I cavities were prepared the same way described before, and after that, all cavity walls (mesial, distal and lingual), excepting the vestibular one, were removed with diamond tips. So that only the vestibular wall remained, which was considered the "flat wall" having the same texture and characteristics as those of the vestibular wall in the Class I model, however, without presenting influence of the C-factor.

On the vestibular wall, methacrylate-based and silorane-based composite blocks were made (after application and light activation of their corresponding adhesive systems, according to the manufacturers' instructions - Figure 1) with dimensions similar to those of the cavities filled in the Class 1 model; that is: $4 \times 3 \times 3 \mathrm{~mm}$. The blocks were light activated with the same Halogen light unit. Both in the Class I model and Flat Surface Model, the specimens were stored in distilled water at $37 \pm 1^{\circ} \mathrm{C}$, for 24 hours.

\section{Microtensile bond strength testing}

The teeth were individually fixed on acrylic plates $(5 \times 5 \times 4 \mathrm{~mm})$ with cyanoacrylate adhesive (Super Bonder, Henkel do Brasil S/A, Itapevi, SP, Brazil). This appliance was fixed in a precision cutter (Imptech PC10, Equilam Ind. e Com. Ltda., Diadema, SP, Brazil) and a high concentration diamond disc (Extec Corp., Enfield, NH, USA) with a cutting speed of $200 \mathrm{rpm}^{22}$ was used to obtain serial sections of the samples, with a distance of 1 $\mathrm{mm}$ between the cuts.

The sections were oriented in such a way as to obtain bonded interfaces between the composite and the vestibular dentin wall, for both cavity models. Thus the dentinal portion of the sticks obtained presented dentinal tubules in a direction parallel to that of the composite surface in the adhesive interface region, irrespective of cavity model. The teeth were removed from the precision cutter and acrylic plate so that the stick-shaped specimens could be selected. Each tooth yielded 2 to 4 stick-shaped specimens of resin composite bonded to enamel.

After 24 hours, a cyanoacrylate-based adhesive was used to fix the specimens, by their extremities, to the microtensile device coupled to a universal test machine (EMIC Equipamentos e Sistemas de Ensaio Ltda., São José dos Pinhais, PR, Brazil). Microtensile was applied at a speed of $0.5 \mathrm{~mm} / \mathrm{min}$. Until the sample ruptured, and the bond strength values were obtained in kilogram-force. The load required to fracture the specimens, in Mega Pascal $(\mathrm{MPa})$, was calculated after measuring the bond area with a digital caliper (Mitutoyo Sulamericana Ltda., Suzano, SP, Brazil).

\section{Fracture pattern analysis}

After the microtensile test, the specimens were immediately observed, by the same operator, under

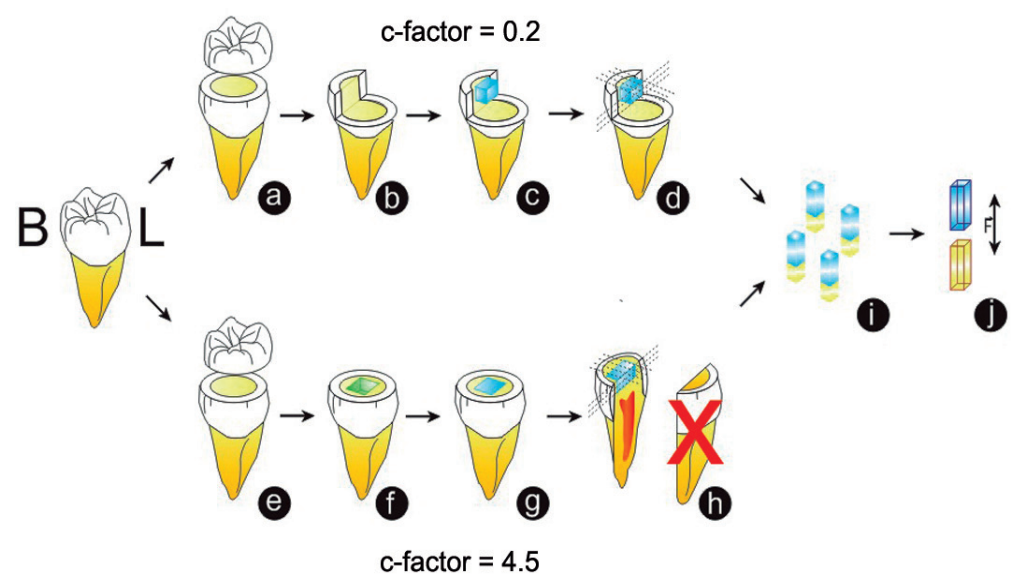

Figure 2- a- Flat Surface cavity model - removal of occlusal enamel; b- Cavity preparation and removal of all the walls, except the vestibular wall (control); c- Resin block fabrication; $d$ - Cuts to obtain specimens for microtensile test; e- Class I cavity model - removal of occlusal enamel; f- Preparation of occlusal cavities; g- Restoration of cavities in a single increment; h- Cuts to obtain specimens for microtensile test; i- Specimens for microtensile test; j - Microtensile test 
a stereomicroscope (EK 35T, Eikonal Equipamentos Ópticos e Científicos Ltda., São Paulo, SP, Brazil), at 20x magnification and the fractures were classified according to one of the following four criteria [adapted from Montes, et al. ${ }^{17}$ (2001)]: Type 1: Adhesive fracture; Type 2: Partially adhesive fracture and partially cohesive in the adhesive; Type 3: cohesive fracture in resin; Type 4: Cohesive fracture in dentin.

Some samples were prepared to illustrate the bond interface in scanning electron microscopy. The samples were fixed on aluminum stubs (Procind Ltda., Piracicaba, SP, Brazil) and sputter-coated (SCD 050 Sputter Coater, Baltec Co., Reading, UK) with gold for $120 \mathrm{~s}$, and then evaluated by scanning electron microscopy (Philips $x \mathrm{l} 30$ scanning electron microscope, FEI Company, Hillsboro, OR, USA) at 500x magnification.
Statistical analysis

The data obtained were tabulated and submitted to the Analysis of Variance and the Tukey Kramer tests, with a level of significance of $5 \%$.

\section{RESULTS}

According to the results, the two-way ANOVA demonstrated statistical difference for the factor "cavity model" $(p=0.0005)$ and interaction between the factors "restorative systems" $X$ "cavity models" $(p<0.05)$.

After application of Tukey's test, it was observed that, for the Flat Surface cavity model, P60 restorative system showed higher bond strength compared with P90 $(p<0.05)$. Also, the bond strength values for $\mathrm{P} 60$ restorative system in Class I cavity model was statistically lower when compared with the Flat Surface cavity model $(p<0.05)$. For

Table 1- Mean bond strength in MPa (standard deviation) considering resin and cavity model

\begin{tabular}{ccccc}
\hline Cavity & \multicolumn{4}{c}{ Restorative System } \\
& $\mathbf{n}$ & $\mathbf{P 6 0}$ & $\mathbf{n}$ & $\mathbf{P 9 0}$ \\
\hline Flat Surface cavity model & 8 & $32.4(8.2)^{\mathrm{Aa}}$ & 10 & $24.4(7.4)^{\mathrm{Ba}}$ \\
Class I cavity model & 10 & $19.0(6.7)^{\mathrm{Ab}}$ & 10 & $19.2(8.4)^{\mathrm{Aa}}$ \\
\hline
\end{tabular}

Means followed by different letters, (capitals in the horizontal and lower case in the vertical) differ among them $(p<0.05)$

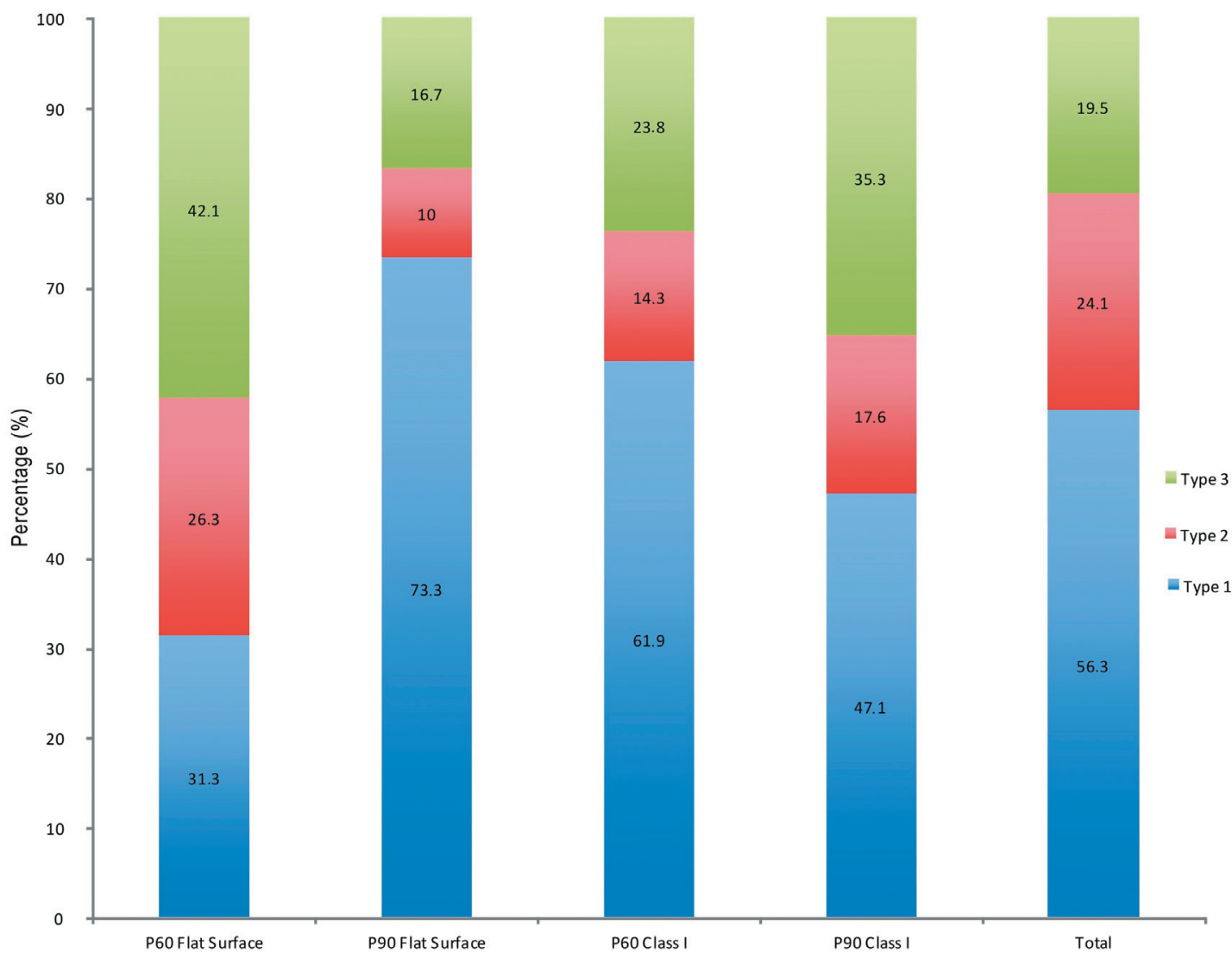

Figure 3- Percentage of fracture patterns. Type 1: Adhesive fracture; Type 2: Partially adhesive fracture and partially cohesive in the adhesive; Type 3: cohesive fracture in resin 


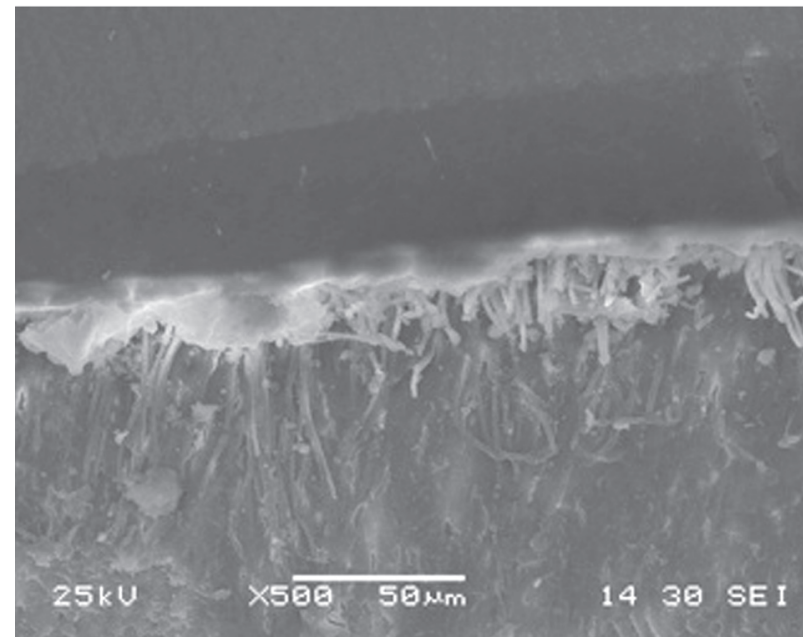

Figure 4- Photomicrograph of vestibular surface of Class I cavity preparation restored with P60/ Adper Single Bond 2 restorative system

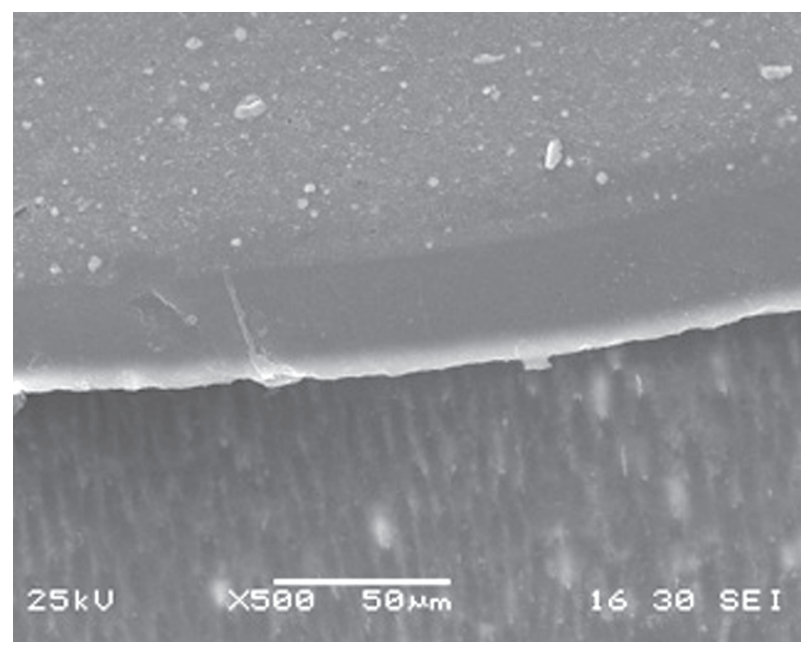

Figure 6- Photomicrograph of vestibular surface of Class I cavity preparation restored with P90/ Silorane Adhesive System

P90 restorative system, the results demonstrated that there was no statistically significant difference between the cavity preparation models (Table 1 ).

Taking into consideration the cavity preparations, it could be observed that for the Flat Surfaces models, the $\mathrm{P} 60$ restorative system obtained statistically higher bond strength than the P90 restorative system, whereas in the Class I cavity model there were no statistically significant differences between $\mathrm{P} 60$ and $\mathrm{P} 90$ restorative systems (Table 1 ).

In Figure 3, the fracture pattern results are presented. It was observed that $56.3 \%$ of the fractures were of Type 1 (adhesive); $24.1 \%$ were of Type 2 (partially adhesive and partially cohesive in adhesive) and $19.5 \%$ of the fractures were of Type 3 (cohesive in resin). No Type 4 fractures occurred and there was no statistically significant difference

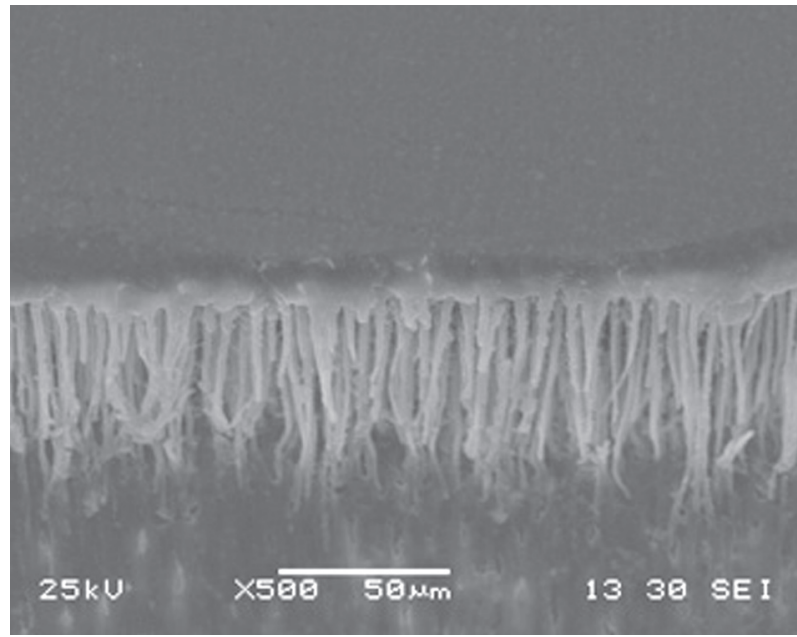

Figure 5- Photomicrograph of flat vestibular surface restored with P60/Adper Single Bond 2 restorative system

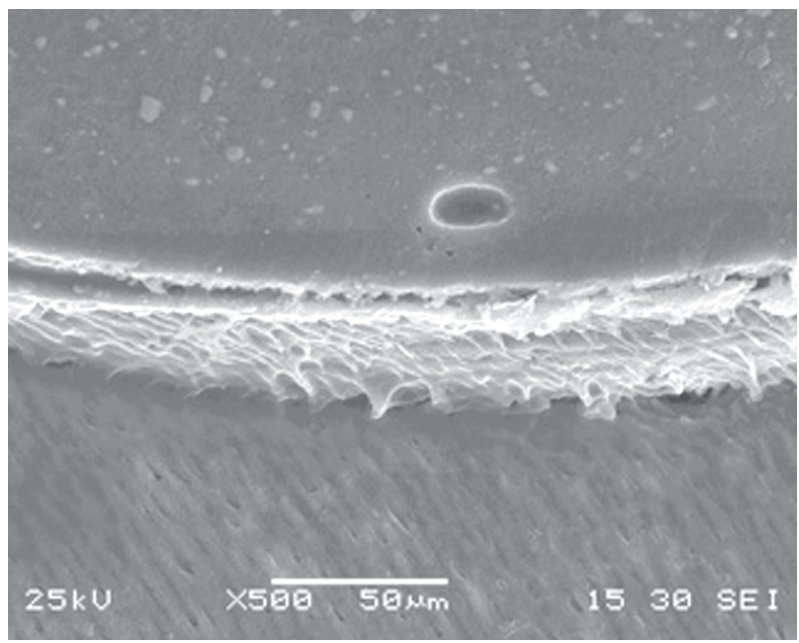

Figure 7- Photomicrograph of flat vestibular surface restored with restored with $\mathrm{P} 90 /$ Silorane Adhesive System

between the fracture patterns $(p=0.0713)$. The interfaces between the different systems and restoratives and cavity models are illustrated in Figures 4 to 7 .

\section{DISCUSSION}

The null hypothesis was rejected since the results showed influence of the restorative systems and the cavity models on bond strength to dentin.

Various methods have been used to measure the influence of contraction stress at the adhesive interface, such as the microleakage test ${ }^{4,28}$ and microtensile bond strength test ${ }^{5-6,14,26}$. In the present study, it may be observed that the bond strength obtained in the Flat Surface cavity model was significantly lower for the P90 composite, when compared with P60 composite. The adhesive system of silorane-based restorative system is a self- 
etching adhesive and it is exclusively for use with this composite ${ }^{11,14,16}$. The Silorane System Adhesive (SSA) is composed of an acidic, hydrophilic onestep primer of the self-etching type (SSA Primer), whose $\mathrm{pH}$ of 2.7 is considered relatively mild that leads to dentin decalcification being resumed to a few hundredths of nanometers ${ }^{16}$. A more viscous and hydrophobic resin adhesive (SSA Bond), must be applied and light activated independently ${ }^{6,25}$. The exact manner in which the two hydrophilic and hydrophobic components bound to each other is unknown ${ }^{16}$. It is known that the primer is composed of a phosphated functional monomer, dimethacrylates (HEMA, Bis-GMA), copolymers of itaconic and acrylic acids, silica and camphorquinone dissolved in a solution of water and alcohol ${ }^{16}$. The high concentration of HEMA is used to prevent separation of the primer phases, but makes it vulnerable to water sorption ${ }^{16}$. In effect, due to the extremely hydrophobic nature of the bond, basically composed of substituted dimethacrylate, TEGDMA, silica, camphorquinone, and to the relatively low concentration of functional monomers ${ }^{12,16}$, there is presence of water exactly between the layers of the primer and bond ${ }^{16}$. As a consequence, the adhesive interface may be considered the weak link of the adhesive system ${ }^{12}$ with reduced bond strength. Also, it has been speculated that the silorane-based primer may be considered a one-step adhesive system, as the primer is light cured ${ }^{21}$. This may justify the low bond strength values of siloranebased primer compared to the two-step adhesive system. It is known that one-step adhesives are more hydrophilic ${ }^{16}$ and have inferior performance regarding bond strength ${ }^{21}$.

In all the groups, there was a higher percentage of adhesive failures, except for Group "P60 Flat", which presented a higher degree of cohesive failures in resin (Figure 3 ). These cohesive failures of the "P60 Flat" group may also be attributed to the adhesive strategy of the conventional systems that use acid etching before application of the adhesive itself, corroborating the results of the microtensile test. Thus, the larger quantity and depth of tags obtained with this type of system ${ }^{24}$ (Figures 4 and 5) may promote deeper micromechanical interlocking ${ }^{6}$, which may generate greater microtensile bond strength ${ }^{24}$ and cohesive failures in resin, as observed in this group, particularly on a flat surface without the influence of the C-factor on the polymerization contraction stresses. The adhesive failures may also be related to the single increment used to restore the cavities, that improved the influence of the $\mathrm{C}$-factor in raising the stress at the restoration/ dentin interface.

It is important to point out that the Flat Surface model served as control group of bond strength without the interference of the $\mathrm{C}$-factor, because in this cavity model there was only one bond surface (area of $12 \mathrm{~mm}^{2}$ ) and five free surfaces (area of $54 \mathrm{~mm}^{2}$ ), characterizing a C-factor of 0.2 , which allows greater possibility of dissipating the stresses generated during polymerization ${ }^{5,8}$.

However, when considering the Class I cavity model, there was significant reduction in the bond strength of $\mathrm{P} 60$ restorative system, in comparison with the Flat Surface cavity model (32.4 X 19.0 MPa, respectively). Although the bond of $\mathrm{P} 60$ restorative systems was sufficient to resist the polymerization stress, the contraction stress decreased the bond strength of this restorative system. This can be attributed to the influence of the C-factor value 4.5, provided by five bonded walls $\left(54 \mathrm{~mm}^{2}\right.$ ) and one free wall $\left(12 \mathrm{~mm}^{2}\right)^{5,8}$. In addition, P60 composite was bulk filled and light activated for 40 seconds, exacerbating the stress raised from polymerization contraction of the methacrylate ${ }^{7}$. The reduction in bond strength of P60 restorative system suggests that it was influenced by the contraction stress generated at the bond interface in the cavity with high cavity factor. Although there was a higher bond strength value on the flat surface, it is suggested that the bond was not sufficient to withstand polymerization contraction. The reduction in bond strength may have important consequences for the longevity of composite restorations whose organic matrix is methacrylate, which confirms the clinical need to use the correct adhesive systems and incremental insertion and light activation techniques $^{8}$ in order to minimize the influence of the C-factor on bond strength ${ }^{2,5,8}$. Maybe, if the incremental insertion were used, the bond strength values of $\mathrm{P} 60$ restorative system in Class I cavity model could be enhanced, since the bulk increment used exacerbated the contraction stress in the interface dentin/restorative system.

On the other hand, the composite derived from silorane (P90) presented no significant reduction in the bond strength due to the cavity model ${ }^{1,7}$. Considering that the insertion/light activation technique was also a bulk increment and immediate light activation for $40 \mathrm{~s}$, one could infer that the C-factor had less influence on this composite, confirming its lower degree of polymerization shrinkage $6,13,18,20,27$. It is suggested that the adhesive system of P90, although leading to lower bond strength means, is not subject to the same stress at the bond interface, since there is a lower degree of volumetric shrinkage of the composite. Another important factor to consider is that the lower degree of polymerization shrinkage may not be reflected as a lower degree of stress generation at the bond interface of the composites ${ }^{3,15,26}$. This occurs, according to Hooke's Law, because the stress generated is a product of the volumetric shrinkage by the modulus of elasticity of the material ${ }^{12,15}$. High 
modulus of elasticity values are related to greater difficulty in dissipating internal stresses during the polymerization reaction. In this sense, the modulus of elasticity of P90 is high when compared with composites derived from methacrylate with similar filler ${ }^{3,13}$.

Therefore, the incremental insertion and light activation techniques that increase the pre-gel phase (phase in which stress is more easily dissipated) $)^{8,17}$ cannot be overlooked. In spite of reduced polymerization contraction being an important factor for the clinical durability of restorations, it does not represent less stress at the bond interface ${ }^{2,15,26}$.

\section{CONCLUSION}

It was concluded that the methacrylate-based composite presented higher bond strength to the flat surface, compared with the silorane-based composite. The use of silorane-based composite led to similar bond strength values compared to the methacrylate-based composite in cavities with high C-factor.

\section{ACKNOWLEDGEMENTS}

This research was partially supported by LNNano - Brazilian Nanotechnology National Laboratory, LME/CNPEM/ABTLUS/MCTI.

\section{REFERENCES}

1- Almeida e Silva JS, Rolla JN, Baratieri LN, Monteiro S Jr. The influence of different placement techniques on the microtensile bond strength of low-shrink silorane composite bonded to Class I cavities. Gen Dent. 2011;59:e233-7.

2- Bagis $\mathrm{YH}$, Baltacioglu IH, Kahyaogullari S. Comparing microleakage and the layering of silorane-based resin composite in wide class II MOD cavities. Oper Dent. 2009;34:578-85.

3- Boaro LC, Gonçalves F, Guimarães TC, Ferracane JL, Versluis A, Braga RR. Polymerization stress, contraction and elastic modulus of current low-contraction restorative composites. Dent Mater. 2010;26:1144-50.

4- Bogra P, Gupta S, Kumar S. Comparative evaluation of microleakage in Class II cavities restored with Ceram $X$ and Filtek P-90: an in vitro study. Contemp Clin Dent. 2012;3:9-14.

5- Choi KK, Ryu GJ, Choi SM, Lee MJ, Park SJ, Ferracane JL. Effects of cavity configuration on composite restoration. Oper Dent. 2004;29:462-9.

6- Duarte Junior S, Phark JH, Varjão FM, Sadan A. Nanoleakage, ultramorphological characteristics, and microtensile bond strengths of a new low-shrinkage composite to dentin after artificial aging. Dent Mater. 2009;25:589-600.

7- El-Sahn NA, El-Kassas DW, El-Damanhoury HM, Fahmy OM, Gomaa H, Platt JA. Effect of C-factor on microtensile bond strengths of low-shrinkage composites. Oper Dent. 2011;36:281-92

8- Ferracane JL. Buonocore Lecture. Placing dental composites - a stressful experience. Oper Dent. 2008;33:247-57.

9- Hahnel S, Henrich A, Bürgers R, Handel G, Rosentritt M. Investigation of mechanical properties of modern dental composites after artificial aging for one year. Oper Dent. 2010;35:412-9.
10- Ilie N, Hickel R. Macro-, micro- and nano-mechanical investigations on silorane and methacrylate-based composites. Dent Mater. 2009;25:810-9.

11- Ivanovas S, Hickel R, Ilie N. How to repair fillings made by silorane-based composites. Clin Oral Invest. 2011;15:915-22.

12- Klautau EB, Carneiro KK, Lobato MF, Machado SM, Silva e Souza MH Jr. Low contraction composite resins: influence on sealing ability in unfavorable C-factor cavities. Braz Oral Res. 2011;25:5-12.

13- Lien W, Vandewalle KS. Physical properties of a new siloranebased restorative system. Dent Mater. 2010;26:337-44.

14- Maneenut C, Sakoolnamarka R, Tyas MJ. The repair potential of resin composite materials. Dent Mater. 2011;27:e20-7.

15- Marchesi G, Breschi L, Antoniolli F, Di Lenarda R, Ferracane

J, Cadenaro M. Contraction stress of low-shrinkage composite materials assessed with different testing systems. Dent Mater. 2010;26:947-53.

16- Mine A, De Munck J, Van Ende A, Cardoso MV, Kuboki T, Yoshida $Y$, et al. TEM characterization of a silorane composite bonded to enamel/dentin. Dent Mater. 2010;26:524-32.

17- Montes MA, Goes MF, Cunha MR, Soares AB. A morphological and tensile bond strength evaluation of an unfilled adhesive with low-viscosity composites and a filled adhesive in one and two coats. J Dent. 2001;29:435-41.

18- Oliveira KM, Consani S, Gonçalves LS, Brandt WC, CcahuanaVásquez RA. Photoelastic evaluation of the effect of composite formulation on polymerization shrinkage stress. Braz Oral Res. 2012;26:202-8.

19- Palin WM, Fleming GJ, Burke FJ, Marquis PM, Randall RC. The influence of short and medium-term water immersion on the hydrolytic stability of novel low-shrink dental composites. Dent Mater. 2005;21:852-63.

20- Papadogiannis D, Kakaboura A, Palaghias G, Eliades G. Setting characteristics and cavity adaptation of low-shrinking resin composites. Dent Mater. 2009;25:1509-16.

21- Pucci CR, Oliveira RS, Caneppele TMF, Torres CRG, Borges AB, Tay FR. Effects of surface treatment, hydration and application method on the bond strength of a silorane adhesive and resin system to dentine. J Dent. 2013;41:278-86.

22- Sadek FT, Cury AH, Monticelli F, Ferrari M, Cardoso PE. The influence of the cutting speed on bond strength and integrity of microtensile specimens. Dent Mat. 2005;21:1144-9.

23- Sampaio RK, Wang L, Carvalho RV, Garcia EJ, Andrade AM, Klein-Júnior CA, et al. Six-month evaluation of a resin/dentin interface created by methacrylate and silorane-based materials. J Appl Oral Sci. 2013;21:80-4.

24- Sarr M, Kane AW, Vreven J, Mine A, Van Landuyt KL, Peumans $M$, et al. Microtensile bond strength and interfacial characterization of 11 contemporary adhesives bonded to bur-cut dentin. Oper Dent. 2010;35:94-104.

25- Sauro S, Pashley DH, Mannocci F, Tay FR, Pilecki P, Sherriff M, et al. Micropermeability of current self-etching and etch-and-rinse adhesives bonded to deep dentine: a comparison study using a double-staining/confocal microscopy technique. Eur J Oral Sci. 2008; 116:184-93.

26- Van Ende A, De Munck J, Mine A, Lambrechts P, Van Meerbeek B. Does a low-shrinking composite induce less stress at the adhesive interface? Dent Mater. 2010;26:215-22.

27- Weinmann W, Thalacker C, Guggenberger R. Siloranes in dental composites. Dent Mater. 2005;21:68-74.

28- Yamazaki PC, Bedran-Russo AK, Pereira PN, Swift EJ Jr. Microleakage evaluation of a new low-shrinkage composite restorative material. Oper Dent. 2006;31:670-6. 